\title{
Coherent DS-SS Receiver Using Exponentially Weighted Despreading Function with Imperfect Power Control in Multipath Rayleigh Fading Environment
}

\author{
Yuejin Huang and \\ yjhuang@eee.hku.hk \\ T. S. Ng \\ tsng@eee.hku.hk \\ Department of Electrical and Electronic Engineering, The University of Hong Kong, \\ Pokfulam Road, Hong Kong. Fax: +85225598738
}

\begin{abstract}
In this paper, a closed-form solution for the average bit error rate (BER) performance of a coherent DS-CDMA system with imperfect power control is derived for a multipath Rayleigh fading channel. The RAKE receiver under consideration employs exponentially weighted despreading function with an adjustable parameter $\gamma$ optimized for multiple access interference rejection in multipath environment. The results indicate that the number of active users supported at a given BER for the case of $\gamma$ tuned to maximize the average signal to interference plus noise ratio $\overline{S I N R}$ is much larger than commonly used rectangular despreading function $(\gamma=0)$. It is shown that imperfect power control affects the irreducible BER for the case of $\gamma=0$. On the other hand, the effect of imperfect power control on BER performance for $\gamma$ tuned to maximize $\overline{S I N R}$ is equivalent to a reduction in the average signal to white noise ratio $\bar{\gamma}_{b}$ and hence system performance can be compensated by increasing the transmitter power.
\end{abstract}

\section{INTRODUCTION}

As is well known, the major limitations in CDMA performance are multipath fading, multiple access interference (MAI) and imperfect power control. In many performance analyses of CDMA systems, the assumptions are made that the transmitted power of each user is perfectly controlled and the waveform of despreading sequence in a receiver is the same as the spreading sequence assigned to a reference user [1-4]. However, in a real system, the power control error always exists irrespective of power control methods being used. The effect of imperfect power control on performance was analyzed in [5] with the maximum diversity order of two. However, a typical DS-CDMA system over a terrestrial channel may operate with $L_{R}$-branch diversity for better performance. Therefore, we are motivated to analyze the performance of a DS-CDMA system with power control error using any order of diversity to combat multipath fading. In this paper, the RAKE receiver using the exponentially weighted despreading function $[6,7]$ with an adjustable parameter $\gamma$ is analyzed and we show that by tuning $\gamma$ to maximize $\overline{\text { SINR }}$, the RAKE receiver performs much better than conventional receiver with fixed $\gamma=0$ in multipath fading environment.

\section{SYSTEM AND CHANNEL MODELS}

A. Transmitter Model

Suppose there are $K$ CDMA users accessing the channel. User $k$ transmits a binary data sequence $b_{k}(t)$ and employs a spreading sequence $a_{k}(t)$ to spread each data bit. The spreading and data sequences for the $k$ th user are given by $a_{k}(t)=\sum_{j=-\infty}^{\infty} a_{j}^{(k)} P_{T_{t}}\left(t-j T_{c}\right)$ and $b_{k}(t)=\sum_{j=-\infty}^{\infty} b_{j}^{(k)} P_{r_{b}}\left(t-j T_{b}\right)$ where $T_{c}$ and $T_{b}$ are the chip and data durations, respectively, and $P_{x}(y)=1$ for $0<\mathrm{y}<\mathrm{x}$ and 0 otherwise. In our study, $b_{j}^{(k)}$ and $a_{j}^{(k)}$ are modeled as independent random variables taking values -1 or +1 with equal probabilities. It is assumed that there are $N$ chips of a spreading sequence in the interval of each data bit $T_{b}$ and the spreading sequence for each user has period equal to $N$. In other words, $T_{b}=N T_{c}$ and $a_{j}^{(k)}=a_{j+N}^{(k)}$ for all $-\infty<j<+\infty$. The transmitted signal for the $k$ th user is

$$
S_{k}(t)=\sqrt{2 P} G_{k} b_{k}(t) a_{k}(t) \cos \left(\omega_{0} t+\theta_{k}\right)
$$

where the transmitted power $P$ and the carrier frequency $\omega_{0}$ are common to all users, and the parameter $\theta_{k}$ is the phase of the $k$ th user. The parameter $G_{k}$ represents the power control error for the $k$ th user and is modeled as a random variable uniformly distributed in $\left[1-\varepsilon_{m}, 1+\varepsilon_{m}\right]$ where $\varepsilon_{m}$ represents the maximum value of power control error for all users. Such a distribution for the parameter $G_{k}$ 
implies a complete lack of knowledge of the power control error and is a least favorable distribution [5].

\section{B. Channel Model}

In our analysis we consider a frequency selective multipath channel for the uplink. The equivalent complex lowpass representation of the channel for the $k$ th user is given by

$$
h_{k}(t)=\sum_{l=0}^{L_{k}-1} \beta_{k l} \delta\left(t-\tau_{k l}\right) e^{j n_{k}}
$$

where random variables $\beta_{k l}, \tau_{k l}$ and $\eta_{k l}$ are the $l$ th path gain, delay and phase, respectively, for the $k$ th user. Furthermore, we assume the following: (i) for different users and paths in each link, the random variables $\left\{\beta_{k l}\right\}$, $\left\{\tau_{k l}\right\}$ and $\left\{\eta_{k l}\right\}$ are all statistically independent; (ii) the random phases $\left\{\eta_{k l}\right\}$ are uniformly distributed over $[0,2 \pi$ ] and the path delays $\left\{\tau_{k l}\right\}$ are uniformly distributed over $\left[0, T_{b}\right]$; (iii) for each user, the path gain $\beta_{k l}$ is a random variable with Rayleigh distribution with $E\left[\beta_{k l}^{2}\right]=2 \rho$ which is independent of $k$ and $l$.

At the central station, all user signals, after passing through their own particular channel, are added together and mixed with AWGN $n(t)$ with two-side power spectral density $N_{0} / 2$. Therefore, the input signal at the central station $r(t)$ can be represented by

$$
\begin{gathered}
r(t)=\sqrt{2 P} \sum_{k=1}^{K} \sum_{l=0}^{L_{k}-1} G_{k} \beta_{k l} b_{k}\left(t-\tau_{k l}\right) a_{k}\left(t-\tau_{k l}\right) \cos \left(\omega_{o} t+\phi_{k l}\right) \\
+n_{c}(t) \cos \omega_{0} t-n_{s}(t) \sin \omega_{0} t
\end{gathered}
$$

where $\phi_{k l}=\theta_{k}+\eta_{k l}-\omega_{0} \tau_{k l}$, and the terms $n_{c}(t)$ and $n_{s}(t)$ are lowpass equivalent components of the AWGN $n(t)$. The random phases $\left\{\phi_{k l}\right\}$ are uniformly distributed over $[0,2 \pi]$. The random variables $\left\{G_{k}\right\},\left\{\beta_{k l}\right\},\left\{\tau_{k l}\right\}$ and $\left\{\phi_{k l}\right\}$ are independent of each other.

\section{Receiver Model}

For BPSK modulation, a single-path matched filter of a RAKE receiver using coherent detection is shown in Fig. 1.

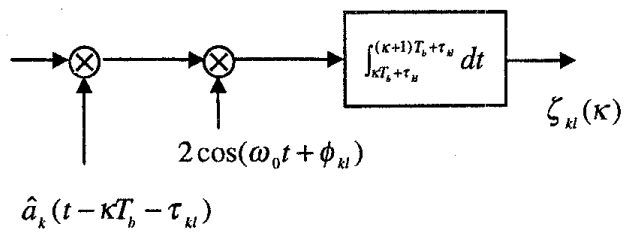

Fig. 1 The structure of a path $l$ matched filter of user's $k$ RAKE receiver.

For MAI rejection, a bank of single-path matched filters, each of which is matched to different paths, have the same impulse response matched to $2 \hat{a}_{k}(t) \cos \left(\omega_{0} t\right) P_{T_{0}}(t)$ where $\hat{a}_{k}(t)$ is the weighted despreading function with details given below. The outputs of all single-path matched filters $\zeta_{k l}(\kappa), l \in\left[0, L_{R}-1\right]$ where $L_{R}$ is the order of diversity, are weighted by the corresponding path gains and then summed to form a single decision variable $\zeta_{k}(\kappa)$. The weighted despreading function of the user $k^{\prime} s$ RAKE receiver can be expressed as $[6,7]$

$$
\hat{a}_{k}(t)=\sum_{j=-\infty}^{\infty} a_{j}^{(k)} w_{j}^{(k)}\left(t-j T_{c} \mid\left\{c_{j}^{(k)}, c_{j+1}^{(k)}\right\}\right) P_{T_{c}}\left(t-j T_{c}\right)
$$

where $c_{j}^{(k)}=a_{j-1}^{(k)} a_{j}^{(k)} ;$ and $w_{j}^{(k)}\left(t \mid\left\{c_{j}^{(k)}, c_{j+1}^{(k)}\right\}\right)$, for $0 \leq t \leq T_{c}$, is the $j$ th chip weighting waveform for the $k$ th receiver conditioned on the status of three consecutive chips $\left\{c_{j}^{(k)}, c_{j+1}^{(k)}\right\}=\left\{a_{j-1}^{(k)} a_{j}^{(k)}, a_{j}^{(k)} a_{j+1}^{(k)}\right\}$. For MAI rejection, we therefore define $[6,7]$

$$
w_{j}^{(k)}\left(t \mid\left\{c_{j}^{(k)}, c_{j+1}^{(k)}\right\}\right)= \begin{cases}m_{1}(t) & \text { if } c_{j}^{(k)}=+1 \text { and } c_{j+1}^{(k)}=+1 \\ m_{2}(t) & \text { if } c_{j}^{(k)}=-1 \text { and } c_{j+1}^{(k)}=-1 \\ m_{3}(t) & \text { if } c_{j}^{(k)}=-1 \text { and } c_{j+1}^{(k)}=+1 \\ m_{4}(t) & \text { if } c_{j}^{(k)}=+1 \text { and } c_{j+1}^{(k)}=-1\end{cases}
$$

with the elements of the chip weighting waveform vector $\left\{m_{1}(t), m_{2}(t), m_{3}(t), m_{4}(t)\right\}$ given by the following:

$$
\begin{aligned}
& m_{1}(t)=e^{-\gamma / 2} P_{T_{c}}(t) \\
& m_{2}(t)=e^{-\gamma t / T_{c}} P_{T_{/ 2}}(t)+e^{-\gamma\left(1-t / T_{c}\right)} P_{T_{c} / 2}\left(t-T_{c} / 2\right) \\
& m_{3}(t)=e^{-\gamma / T_{c}} P_{T_{c} / 2}(t)+e^{-\gamma / 2} P_{T_{t / 2}}\left(t-T_{c} / 2\right) \\
& m_{4}(t)=e^{-\gamma / 2} P_{T_{c} / 2}(t)+e^{-\gamma\left(1-t / T_{c}\right)} P_{T_{c} / 2}\left(t-T_{c} / 2\right)
\end{aligned}
$$

where $\gamma \in[0, \infty)$ is a parameter of the chip weighting waveforms. When $\gamma=0$ in (6), the chip weighting waveforms $m_{p}(t)$ for all $p \in[1,2,3,4]$ reduce to the rectangular pulse $P_{\tau_{c}}(t)$. The chip weighting waveforms are shown in Fig. 2 of [6].

\section{SYSTEM PERFORMANCE}

We arbitrarily choose the $i$ th user as the reference user and analyze the performance of the RAKE receiver with coherent detection for data symbol $b_{\lambda}^{(i)}$. After the $l$ th path matched filter which has the impulse response matched to $2 \hat{a}_{i}(t) \cos \left(\omega_{0} t\right) P_{\tau_{b}}(t)$, the conditional output random variable, denoted by $\zeta_{i l}(\lambda)$, can be expressed as

$\zeta_{i l}(\lambda)=\int_{\lambda T_{b}+\tau_{o}}^{(\lambda+1) T_{b}+\tau_{i l}}\left\{2 r(t) \hat{a}_{i}\left(t-\lambda T_{b}-\tau_{i l}\right) \cos \left(\omega_{v} t+\phi_{i l}\right)\right\} d t(7)$

where $\cos \left(\omega_{0} t+\phi_{i l}\right)$ is the coherent carrier reference and $\hat{a}_{i}\left(t-\lambda T_{b}-\tau_{i l}\right)$ is the weighted despreading function for the $\lambda$ th bit of the $l$ th path signal of the reference user. Since the carrier frequency $f_{0}$ is much larger than $T_{b}^{-1}$ in practical systems, the double-frequency terms in (7) is ignored in our analysis. Then (7) is reduced to

$$
\zeta_{i l}(\lambda)=S_{i l}(\lambda)+N_{i l}(\lambda)+\sum_{\substack{k=1 \\ k \neq i}}^{K} \sum_{n=0}^{L_{k}-1} Y_{k, n, l}+\sum_{\substack{n=0 \\ n \neq i}}^{L_{p}-1} Y_{i, n, l}
$$


where the first, second, third, and forth components are the desired, AWGN, MAI, and self-generated multipath interference components which are described in detail below.

Using (5), (6) and (8), the desired signal term of the RAKE receiver $S_{i}(\lambda)$, which is the weighted sum of the outputs of the single-path matched filters, is given by [7]

$$
\begin{aligned}
& S_{i}(\lambda)=\sum_{i=0}^{I_{k}-1} \beta_{i t} S_{i l}(\lambda) \\
& =b_{\lambda}^{(i)} \sqrt{2 P} G_{i}\left[2 \hat{N}_{i} T_{c}\left(1-e^{-\gamma / 2}\right) / \gamma+\left(N-\hat{N}_{i}\right) T_{i} e^{-\gamma / 2}\right]_{i=0}^{L_{k}-1} \beta_{i t}^{2} .
\end{aligned}
$$

where $\hat{N}_{i}$ is a random variable which represents the number of times of occurrence that $c_{j}^{(i)}=-1$ for all $j \in[0, N-1] . N_{i l}(\lambda)$ in (8) is the $l$ th path AWGN term and is given by

$$
N_{i t}(\lambda)=\int_{0}^{T_{i}}\left[n_{c}(t) \cos \phi_{i l}-n_{s}(t) \sin \phi_{i l}\right] \hat{a}_{i}(t) d t
$$

The total AWGN in the decision variable of the $i$ th RAKE receiver, denoted by $N_{i}(\lambda)$, can be expressed as

$$
N_{i}(\lambda)=\sum_{l=0}^{L_{\mathrm{p}}-1} \beta_{i l} N_{i l}(\lambda) \text {. }
$$

Thus the variance of the total AWGN $N_{i}(\lambda)$ of the $i$ th RAKE receiver, conditioned on $\left\{\beta_{i l}\right\}$ and $\left\{c_{j}^{(i)}\right\}$, and denoted by $\sigma_{\left.N \| \beta_{n}, c^{(t)}\right)}^{2}$, is given by [7]

$$
\sigma_{\left.N k \beta_{i}, e_{i}^{(1)}\right)}^{2}=N_{0}\left[\hat{N}_{i} T_{c}\left(1-e^{-\gamma}\right) / \gamma+\left(N-\hat{N}_{i}\right) T_{c} e^{-\gamma}\right]_{i=0}^{L_{x}-1} \beta_{i l}^{2} \text {. }
$$

The third term in (8) is the MAI term of the $l$ th singlepath matched filter of the $i$ th RAKE receiver and $Y_{k, n, l}$ can be expressed as

$$
Y_{k, n, l}=\sqrt{2 P} G_{k} \beta_{k n} \cos \left(\phi_{i l}-\phi_{k n}\right)\left[b_{\lambda-1}^{(k)} R_{i k}\left(\tau_{n l}^{(k, j)}\right)+b_{\lambda}^{(k)} \hat{R}_{i k}\left(\tau_{n l}^{(k, j)}\right)\right]
$$

where $\tau_{n l}^{(k, i)}=\tau_{k n}-\tau_{i l} \quad, \quad R_{i k}(\tau)=\int_{0}^{\tau} a_{k}(t-\tau) \hat{a}_{i}(t) d t \quad$ and $\hat{R}_{i k}(\tau)=\int_{\tau}^{T_{i}} a_{k}(t-\tau) \hat{a}_{i}(t) d t$. The total MAI of the $i$ th RAKE receiver $I_{i, k}$, which is the weighted sum of the MAI terms of the single-path matched filters, may be expressed as

$$
I_{i, k}=\sum_{i=0}^{L_{n}-1} \beta_{i l} \sum_{\substack{k=1 \\ k \neq i}}^{K} \sum_{n=0}^{L_{n}-1} Y_{k, n, l}
$$

The variance of the total MAI $I_{i, k}$, denoted by $\sigma_{M\left\{\beta_{i} e_{j}^{(i)}\right\}}^{2}$ and conditioned on $\left\{\beta_{i l}\right\}$ and $\left\{c_{j}^{(i)}\right\}$, is then given by [7]

$$
\begin{aligned}
& \sigma_{M\left\langle\beta_{i l}, \varepsilon_{t}^{(i)}\right\}}^{2} \\
& =2 \rho P L_{p}(K-1)\left(1+\frac{\varepsilon_{m}^{2}}{3}\right)\left(\frac{N T_{c}^{2}}{\gamma^{2}} e^{-\gamma}\right) \Xi^{(e)}\left(\Gamma^{\left(c_{j}^{(k)}\right)}, \gamma\right) \sum_{l=0}^{L_{k}-1} \beta_{i l}^{2} .
\end{aligned}
$$

where

$$
\begin{aligned}
& \Xi^{(e)}\left(\Gamma^{\left(c_{j}^{(i)}\right)}, \gamma\right)=\frac{1}{N}\left\{\Gamma_{\{-1,-\{,-1)}^{(i)}\left[4+\frac{12}{\gamma}-\frac{16}{\gamma} e^{\gamma / 2}+\frac{4}{\gamma} e^{\gamma}\right]\right. \\
& +\left(\Gamma_{\{-1,1,1\}}^{(i)}+\Gamma_{\{1,-1,-1\}}^{(i)}\right)\left[\frac{5}{2}-\frac{\gamma}{4}+\frac{\gamma^{2}}{24}+\frac{19}{2 \gamma}+e^{\gamma / 2}-\frac{12}{\gamma} e^{\gamma / 2}+\frac{5}{2 \gamma} e^{\gamma}\right] \\
& +\left(\Gamma_{\{-1,1,1)}^{(i)}+\Gamma_{(1,1,-1\}}^{(i)}\right)\left[-\frac{3}{2}-\frac{3}{4} \gamma+\frac{19}{24} \gamma^{2}-\frac{1}{2 \gamma}+e^{\gamma / 2}+\frac{e^{\gamma}}{2 \gamma}\right] \\
& +\Gamma_{\{-1,1,-1)}^{(i)}\left[-3-\frac{3}{2} \gamma+\frac{7}{12} \gamma^{2}-\frac{1}{\gamma}+2 e^{\gamma / 2}+\frac{e^{\gamma}}{\gamma}\right] \\
& \left.+\Gamma_{\{1,-1,1)}^{(i)}\left[1-\frac{\gamma}{2}+\frac{\gamma^{2}}{12}+\frac{7}{\gamma}+2 e^{\gamma / 2}-\frac{8}{\gamma} e^{\gamma / 2}+\frac{e^{\gamma}}{\gamma}\right]+\gamma^{2} \Gamma_{(1,1,1)}^{(i)}\right\}
\end{aligned}
$$

$\Gamma_{\left\{v_{1}, v_{2}, v_{3}\right\}}^{(i)}$ is the number of times of occurrence that $\left\{c_{j-1}^{(i)}, c_{j}^{(i)}, c_{j+1}^{(i)}\right\}=\left\{v_{1}, v_{2}, v_{3}\right\}$ for all $j \in[0, N-1]$ in the $i$ th weighted despreading sequence and each element of $\left\{v_{1}, v_{2}, v_{3}\right\}$ takes values +1 or -1 with equal probabilities. It is clear that $\sum_{\left\{v_{1}, v_{2}, v_{3}\right\}} \Gamma_{\left\{v_{1}, v_{2}, v_{3}\right\}}^{(i)}=N$.

The fourth term in (8) is the self-generated multipath interference term of the $l$ th single-path matched filter. However, the total self-interference of the $i$ th RAKE receiver does not have a significant effect on performance comparison because of the fact that the self-generated interference will have much less power than the MAI when the number of active users $K \gg>1$. Therefore, for simplicity, we ignore this term in our analysis.

By definition, the total conditional signal to interference plus noise ratio $S I N R_{i}$ of the decision variable of the reference RAKE receiver may be expressed in the form

$$
\operatorname{SINR}_{\mathrm{i}}=\left(\frac{\sigma_{\left.N \mid \beta_{u}, c_{j}^{(i)}\right\}}^{2}}{S_{i}^{2}(\lambda)}+\frac{\sigma_{M \mid\left\{\beta_{u}, c_{j}^{(i)}\right\}}^{2}}{S_{i}^{2}(\lambda)}\right)^{-1}
$$

where $S_{i}(\lambda), \sigma_{N\left\{\beta_{u}: c_{j}^{(i t}\right\}}^{2}$ and $\sigma_{\left.M \| \beta_{u}, c_{j}^{(i)}\right\}}^{2}$ are given by (9), (12) and (15), respectively.

Because the MAI is the sum of many independent couser signals, it can be modeled as conditional zero-mean Gaussian process. Using this approach the conditional error probability $P_{\left.e \nvdash \beta_{i}\right\} G_{t}}^{(i)}$, conditioned on $\left\{\beta_{i l}\right\}, G_{i}$ and $\left\{c_{j}^{(i)}\right\}$, is given by

$$
P_{\left.e \nvdash \beta_{n}\right\}, G_{i}}^{(i)}=Q\left(\sqrt{\operatorname{SINR}_{i}}\right)
$$

where

$$
Q(x)=\frac{1}{\sqrt{2 \pi}} \int_{x}^{\infty} e^{-t^{2} / 2} d t .
$$

It is well known that the RAKE receiver under consideration is equivalent to a receiver with maximal ratio combiner [8]. Since it is assumed that $E\left[\beta_{i t}^{2}\right]=2 \rho$ for all paths and users, the conditional error probability $P_{e \mid G}^{(i)}$, conditioned on $G_{i}$ and $\left\{c_{j}^{(i)}\right\}$, is then given by (eq.(7.4.15) of [8] ) 


$$
P_{e \mid G_{,}}^{(i)}=\left(\frac{1-\mu}{2}\right)^{L_{k}} \sum_{k=0}^{L_{k}-1}\left(\begin{array}{c}
L_{R}-1+k \\
k
\end{array}\right)\left(\frac{1+\mu}{2}\right)^{k}
$$

where

$$
\mu=\sqrt{R_{c} /\left(1+R_{c}\right)} \text { and } R_{c}=[\overline{S I N R}]^{2} G_{i}^{2}
$$

and $\overline{S I N R}$ is the average signal to interference plus noise ratio per channel, given by

$$
\begin{aligned}
& \overline{S I N R}=\left\{\frac{\gamma\left[\chi\left(1-e^{-\gamma}\right)+\gamma(1-\chi) e^{-\gamma}\right]}{\bar{\gamma}_{b}\left[2 \chi\left(1-e^{-\gamma / 2}\right)+\gamma(1-\chi) e^{-\gamma / 2}\right]^{2}}\right. \\
& \left.+\frac{\left(K L_{p}-1\right)\left(1+\varepsilon_{m}^{2} / 3\right) \Xi^{(e)}\left(\Gamma^{\left(c_{i}^{(i)}\right)}, \gamma\right)}{N\left[2 \chi\left(e^{\gamma / 2}-1\right)+\gamma(1-\chi)\right]^{2}}\right\}^{-1 / 2}
\end{aligned}
$$

with $\chi=\hat{N}_{i} / N, \bar{\gamma}_{b}=\bar{E}_{b} / N_{0}$ and $\bar{E}_{b}=2 \rho P T_{b}$. In order to remove the conditional probability on $G_{i}$ in (20), and obtain $P_{e}^{(i)}$, we evaluate the integral

$$
P_{e}^{(i)}=\frac{1}{2 \varepsilon_{m}} \int_{1-\varepsilon_{*}}^{1+\varepsilon_{m}} P_{e \mid G_{i}}^{(i)} d G_{i} .
$$

According to Appendix A of [7], a closed-form solution for (23) is given by

$$
P_{e}^{(i)}=\frac{1}{2}-\sum_{d=1}^{L_{A}} \frac{\left(\begin{array}{c}
2 d-2 \\
2^{2 d} \varepsilon_{m}
\end{array}\right)}{\operatorname{SINR}(2 d-3)}\left\{\left(b^{2}+1\right)^{\frac{3-2 d}{2}}-\left(a^{2}+1\right)^{\frac{3-2 d}{2}}\right\}
$$

where $a=\left(1+\varepsilon_{m}\right) \overline{\operatorname{SINR}}$ and $b=\left(1-\varepsilon_{m}\right) \overline{\operatorname{SINR}}$. Eq.(24) will enable us to compute the BER for coherent reception at a given set of system parameters.

\section{NUMERICAL RESULTS}

In this section, we present numerical results on the BER performance of a coherent DS-CDMA system with power control error using RAKE reception over a multipath Rayleigh fading channel. We demonstrate the improvement on performance using the proposed weighted despreading function and the degradation of performance due to imperfect power control. In the following, it is assumed that the average fading power $\rho$ is perfectly estimated and the $i$ th user's spreading sequence is a random binary sequence with length $N=255$. In this sequence, $\hat{N}_{i}=134, \Gamma_{\{-1,-1,-1\}}^{(i)}=33, \Gamma_{\{-1,-1,1\}}^{(i)}+\Gamma_{\{1,-1,-1\}}^{(i)}=70$, $\Gamma_{\{-1,1,1\}}^{(i)}+\Gamma_{\{1,1,-1\}}^{(i)}=60, \quad \Gamma_{\{-1,1,-1\}}^{(i)}=36, \quad \Gamma_{\{1,-1,1\}}^{(i)}=31, \quad$ and $\Gamma_{\{1,1,1\}}^{(i)}=25$. We use (24) to compute the probabilities of error at a given set of system parameters.

Fig. 2 shows the BER versus the average signal to white noise ratio $\bar{\gamma}_{b}$ with diversity order $L_{R}$ varies from 1 to 4 .

BER

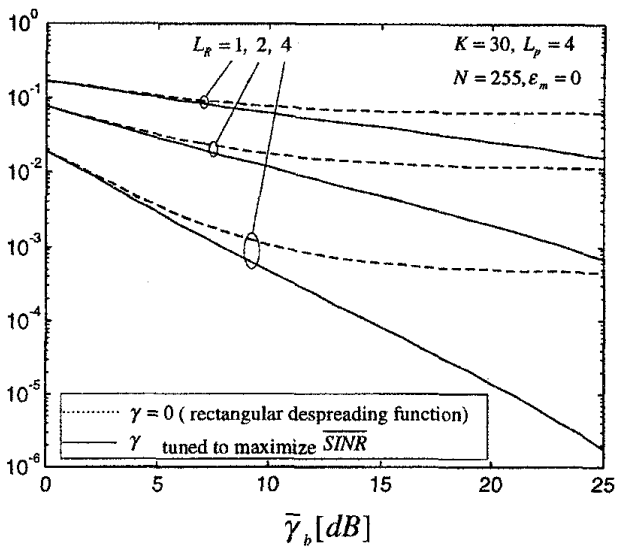

Fig. 2 BER's versus $\bar{\gamma}_{b}$ for RAKE receiver with different order of diversity.

The dashed and solid lines in the figure correspond to the cases of $\gamma=0$ and $\gamma$ tuned to maximize $\overrightarrow{S I N R}$ where $\overline{\text { SINR }}$ is the average signal to interference plus noise ratio given in (22). The case of $\gamma=0$ represents the case that the despreading sequence in the receiver consists of rectangular pulses and is identical to the spreading sequence of the reference user. The improvement on performance is obvious in multipath fadding environment when $\gamma$ is tuned to maximize $\overline{\operatorname{SINR}}$. As expected, the BER decreases as the order of diversity $L_{R}$ increases for both cases of $\gamma=0$ and $\gamma$ tuned to maximize $\overline{S I N R}$.

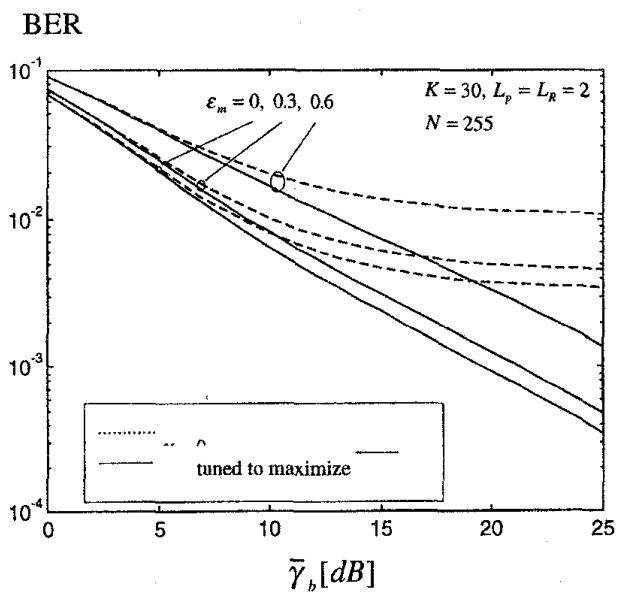

Fig. 3 BER's versus $\bar{\gamma}_{b}$ for RAKE receiver with the muximum power control error $\mathcal{E}_{m}$ as a parameter.

Fig. 3 shows the BER performance versus $\bar{\gamma}_{b}$ with the maximum value of power control error $\varepsilon_{m}$ as a parameter. It is clear that the BER performance degrades for both cases of $\gamma=0$ and $\gamma$ tuned to maximize $\overline{\text { SINR }}$ as $\varepsilon_{m}$ increases. In relative high $\bar{\gamma}_{b}(\geq 15 d B), \varepsilon_{n}$ affects the 
irreducible error probability (or floor of error probability) for the case of $\gamma=0$. As a result, the degradation of system performance can not be compensated by simply increasing $\bar{\gamma}_{b}$. However, for the case of $\gamma$ tuned to maximize $\overline{\text { SINR }}$, the effect on performance due to imperfect power control is equivalent to a reduction of $\vec{\gamma}_{b}$ so that the degradation of system performance can be compensated by increasing $\bar{\gamma}_{b}$. For example, as $\varepsilon_{m}$ increases from 0 to 0.3 , the penalty of $\bar{\gamma}_{b}$ is about $1.25 \mathrm{~dB}$ when $\bar{\gamma}_{b} \geq 10 \mathrm{~dB}$.

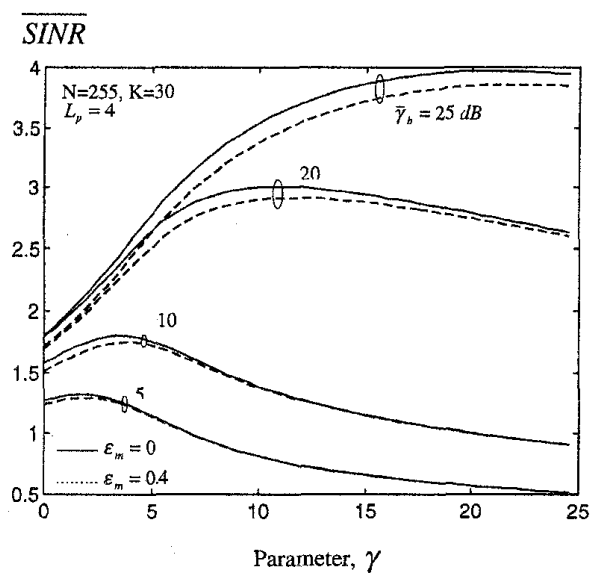

Fig. $4 \overrightarrow{S I N R}$ versus the parameter $\gamma$ for various values of $\bar{\gamma}_{h}$ when $\varepsilon_{m}=0$ and $\varepsilon_{m}=0.6$.

In Fig. 4, the average signal to interference plus noise ratio $\overline{\text { SINR }}$ is plotted as a function of $\gamma$ for various values of $\vec{\gamma}_{b}$. The solid and dashed curves correspond to the cases of $\varepsilon_{m}=0$ and $\varepsilon_{m}=0.6$, respectively. One can see that the effect on $\overline{\text { SINR }}$ due to imperfect power control is not obvious. Based on the results shown in Fig. 3, we can see that the degree of $\overline{\text { SINR }}$ reduction relative to that of BER performance degradation due to imperfect power control is very small.

\section{v. CONCLUSIONS}

In this paper, the BER performance of a coherent DSCDMA system with power control error using RAKE reception over a multipath Rayleigh fading channel has been evaluated and a closed-form solution for the BER performance has been obtained. For MAI rejection, the RAKE receiver employs the exponentially weighted despreading function. Numerical results show that tremendous improvement on performance can be achieved using both multipath diversity and the proposed weighted despreading function in the receivers. Imperfect power control for the case of $\gamma=0$ increases the irreducible error probability (or floor of error probability) in relative high average signal to noise ratio $\bar{\gamma}_{b}$. On the other hand, the penalty on performance with power control error for the case of $\gamma$ tuned to maximize $\overline{\text { SINR }}$ is equivalent to a reduction of $\bar{\gamma}_{b}$ at a given BER in a DS-CDMA system. Therefore, when $\gamma$ is tuned to maximize $\overline{S I N R}$, the degradation of performance due to imperfect power control can be compensated by increasing $\bar{\gamma}_{b}$. We also show that the effect due to imperfect power control on the BER performance is significant while that on the maximum value of $\overline{\operatorname{SINR}}$ obtained by tuning $\gamma$ is rather insignificant

\section{ACKNOWLEDGMENT}

This work was supported by the Research Grants Council of Hong Kong and the CRCG of The University of Hong Kong.

\section{REFERENCES}

[1] M. B. Pursley, "Performance Evaluation for PhaseCoded Spread-Spectrum Multiple-Access Communication - Part I: System Analysis," IEEE Trans. Commun., vol. COM-25, pp. 795-799, Aug. 1977.

[2] M. Kavehrad and B. Ramamurthi, "Direct-Sequence Spread Spectrum with DPSK Modulation and Diversity for Indoor Wireless Communications," IEEE Trans. Commun., vol. COM-35, No. 2, pp. 224-236, Feb. 1987

[3] J. Wang, M. Moeneclaey and L. B. Milstein, "DSCDMA with Predetection Diversity for Indoor Radio Communications," IEEE Trans. Commun., vol. 42, No. 2/3/4, pp. 1929-1938, Feb./Mar./Apr. 1994.

[4] T. Eng and L. B. Milstein, "Comparison of Hybrid FDMA/CDMA Systems in Frequency Selective Rayleigh Fading," IEEE J. Select. Areas Commun., vol. 12, No. 5, pp. 938-951, June 1994.

[5] B. R. Vojcic, R. L. Pickholtz, and L. B. Milstein, "Performance of DS-CDMA with Imperfect Power Control Operating Over a Low Earth Orbiting Satellite Link," IEEE J. Select. Areas Commun., vol. 12, No. 4, pp. 560-567, May 1994.

[6] Y. Huang and T.S. Ng, "Effects of Weighted Despreading Sequence on CDMA Performance," in Proceedings of IEEE International Symposium on Personal, Indoor and Mobile Radio Communications., Helsinki, Finland, Sept. 1-4, 1997 , vol. 1, pp. 226-230.

[7] Y. Huang and T.S. Ng, "DS-CDMA with Power Control Error Using Weighted Despreading Sequences Over a multipath Rayleigh Fading Channel," to appear in IEEE Trans. Vehic. Tech..

[8] J. G. Proakis, Digital Communications. New York: McGraw-Hill, 1989. 